\title{
Spontaneous radiation of a finite-size dipole emitter in hyperbolic media
}

\author{
Alexander N. Poddubny, ${ }^{1,2}$ Pavel A. Belov, ${ }^{1,3}$ and Yuri S. Kivshar ${ }^{1,4}$ \\ ${ }^{1}$ Department of Photonics and Optoinformatics, St. Petersburg University for Information Technology, \\ Mechanics and Optics (ITMO), St. Petersburg RU-197101, Russia \\ ${ }^{2}$ Ioffe Physical-Technical Institute of the Russian Academy of Science, St. Petersburg RU-194021, Russia \\ ${ }^{3}$ School of Electronic Engineering and Computer Science, Queen Mary University of London, Mile End Road, London E1 4NS, UK \\ ${ }^{4}$ Nonlinear Physics Center and Center for Ultrahigh-bandwidth Devices for Optical Systems (CUDOS), Research School of Physics and \\ Engineering, Australian National University, Canberra ACT 0200, Australia
}

(Received 28 May 2011; published 8 August 2011)

\begin{abstract}
We study the radiative decay and Purcell effect for a finite-size dipole emitter placed in a homogeneous uniaxial medium. We demonstrate that the radiative rate is strongly enhanced when the signs of the medium longitudinal and transverse dielectric constants are opposite, and that the isofrequency contour corresponds to a hyperbolic medium. We reveal that the Purcell enhancement factor remains finite even in the absence of losses and that it depends on the emitter size.
\end{abstract}

DOI: 10.1103/PhysRevA.84.023807

PACS number(s): 42.50.-p, 74.25.Gz, 78.70.-g

\section{INTRODUCTION}

The Purcell effect is the enhancement of the spontaneous emission for a source placed in a resonant cavity as compared to that in vacuum [1]. Engineering of the radiative lifetime is now extensively studied in a variety of different systems including metallic particles [2-5], microcavities [6-8], and metamaterials [9-13].

The huge Purcell factor for a point dipole embedded in the so-called hyperbolic medium has been reported in Ref. [14]. This system, namely, a uniaxial medium where the transverse $\varepsilon_{x x}=\varepsilon_{y y} \equiv \varepsilon_{\perp}$ and longitudinal $\varepsilon_{z z}=\varepsilon_{\|}$dielectric constants have opposite signs, is characterized by hyperbolic isofrequency contours in wave-vector space [15-17] (see also insets in Fig. 1). Wave propagation and refraction in the hyperbolic medium reveals its unusual optical properties as compared to the uniaxial medium with the ellipsoidal isofrequency surface [15,18-20]. The radiative decay rate of the point dipole in such a medium diverges and it remains finite only due to the inevitable losses [11-13].

In this work, we consider a finite-size light source such as a quantum dot placed in a homogeneous uniaxial medium with $\varepsilon_{\|} \varepsilon_{\perp}<0$. We demonstrate that, for the spatially distributed source, the radiative rate does not diverge even for vanishing losses but instead depends strongly on the source size. The maximum enhancement of the Purcell factor can be roughly estimated as $(\lambda / a)^{3}$, where $\lambda$ is the wavelength of light in vacuum and $a$ is the characteristic size of the source.

Importantly, the hyperbolic medium employed in our calculations presented below is not only a hypothetic theoretical model. In the simplest case, it appears as an effective medium in the theories describing the averaged characteristics of the layered structure composed of alternating dielectric and metallic layers [21-23] or a mesh created by metallic wires or a plasmonic crystal of nanorods [24-27].

The paper is organized as follows: In Sec. II we introduce our model and effective medium and outline the calculation technique. The results of our calculations are summarized in Sec. III, and Sec. IV concludes the paper. Some specific details of calculations are given in the Appendix A.

\section{MODEL AND EFFECTIVE MEDIUM}

We consider a spherical light source (e.g., a quantum dot) embedded into an anisotropic homogeneous medium characterized by the dielectric tensor $\hat{\varepsilon}$. The equation for the electric field reads

$$
\operatorname{rot} \operatorname{rot} \boldsymbol{E}=q_{0}^{2} \boldsymbol{D},
$$

where $q_{0}=\omega / c$ with $\omega$ being the wave frequency and $c$ being the speed of light in vacuum. The displacement vector includes the background contribution and resonant polarization of the emitter $\boldsymbol{P}$ :

$$
\boldsymbol{D}=\hat{\varepsilon} \boldsymbol{E}+4 \pi \boldsymbol{P},
$$

and the nonzero components of the complex dielectric tensor are

$$
\varepsilon_{x x}=\varepsilon_{y y}=\varepsilon_{\perp}^{\prime}+i \varepsilon_{\perp}^{\prime \prime}, \quad \varepsilon_{z z}=\varepsilon_{\|}^{\prime}+i \varepsilon_{\|}^{\prime \prime} .
$$

We assume that the dielectric tensor describes the hyperbolic medium with $\varepsilon_{\|}^{\prime} \varepsilon_{\perp}^{\prime}<0$. Importantly, such a medium appears as an effective medium in the homogenization of the artificial photonic structures-metamaterials. The simplest example is the layered structure consisting of alternating dielectric and metallic slabs [21-23]. Its effective parameters are given by [28]

$$
\varepsilon_{\perp}=\frac{a \varepsilon_{a}+b \varepsilon_{b}}{a+b}, \quad \frac{1}{\varepsilon_{\|}}=\frac{1}{a+b}\left(\frac{a}{\varepsilon_{a}}+\frac{b}{\varepsilon_{b}}\right),
$$

where $a, b$ and $\varepsilon_{a}, \varepsilon_{b}$ are thicknesses and dielectric constants of the constituting layers, respectively. By properly adjusting the values of these parameters one can achieve the hyperbolic medium regime when $\varepsilon_{\|} \varepsilon_{\perp}<0$. Another metamaterial that has been realized is the structure created from a mesh of metallic wires [24-27]. In this latter case one should also take into account the effects of strong spatial dispersion $[29,30]$ and the excitation of plasmons [31-33]. We note, that the hyperbolic regime can be also attained in a plasma subjected to a strong static magnetic field [34-36]. 

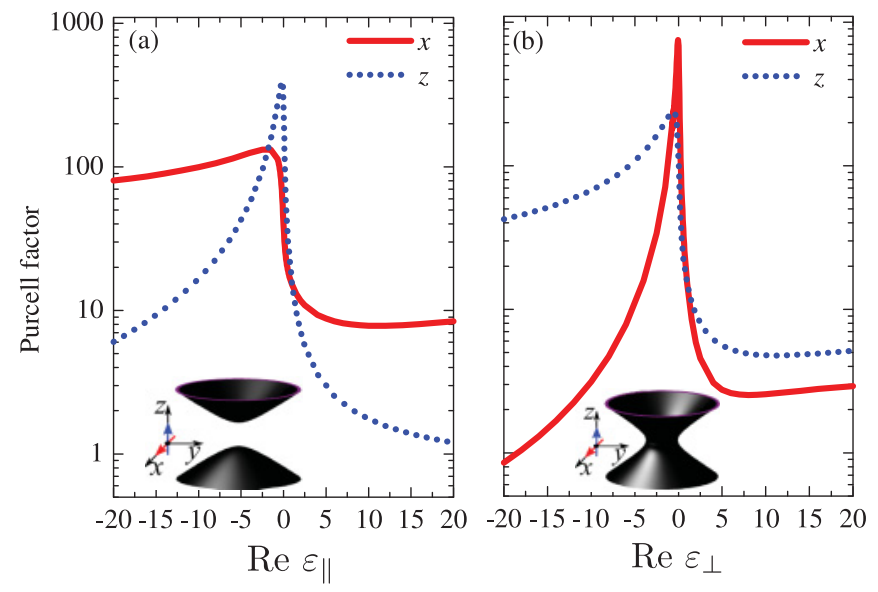

FIG. 1. (Color online) Purcell factor relative to vacuum as a function of (a) $\varepsilon_{\|}^{\prime}$ for $\varepsilon_{\perp}^{\prime}=1$ and (b) $\varepsilon_{\perp}^{\prime}$ for $\varepsilon_{\|}^{\prime}=1$. Solid and dashed lines correspond to the dipole oriented along the $x$ and $z$ axes, respectively. The insets show schematically the isofrequency surfaces in $\boldsymbol{k}$ space for $\varepsilon_{\perp}^{\prime} \varepsilon_{\|}^{\prime}<0$. The calculation was performed for $q_{0} a=0.1$ and $\varepsilon_{\|}^{\prime \prime}=\varepsilon_{\perp}^{\prime \prime}=0.1$.

We write the phenomenological material equation for the polarization as $[37,38]$

$$
\boldsymbol{P}=\frac{d^{2} \Phi(r)}{\hbar\left(\omega_{0}-\omega\right)} \int d^{3} r^{\prime} \Phi\left(r^{\prime}\right) \boldsymbol{E}\left(\boldsymbol{r}^{\prime}\right) .
$$

Here, $\omega_{0}$ is the resonance frequency, $d$ is the effective matrix element of the dipole moment of the emitter, and the function $\Phi(r)$ characterizes the spatial distribution of the emitter polarization. In what follows, we use $\Phi(r)$ in the simple Gaussian form

$$
\Phi(r)=\frac{\sqrt{2} \exp \left[-r^{2} /\left(2 a^{2}\right)\right]}{4 \pi^{3 / 2} a^{3}},
$$

so that $\int d^{3} r \Phi(r)=1$. Equation (5) is similar to the material relation for the excitonic polarization of the semiconductor quantum dot; see Refs. [37-41]. In this case $\Phi(r)$ is the excitonic envelope function and the factor $d^{2}$ is proportional to the longitudinal-transverse splitting $\omega_{\mathrm{LT}}[39]$.

The radiative lifetime $\tau$ is related to the complex eigenfrequency $\omega$ of the homogenous system of Eqs. (1)-(5) as $[38,40,41]$

$$
\frac{1}{\tau}=-2 \operatorname{Im} \omega
$$

To find $\tau$ we apply the Fourier transform

$$
\boldsymbol{E}(\boldsymbol{r})=\int \frac{d^{3} k}{(2 \pi)^{3}} \boldsymbol{E}_{\boldsymbol{k}} e^{i \boldsymbol{k} \cdot \boldsymbol{r}}
$$

and obtain

$$
\begin{aligned}
& {\left[q_{0}^{2} \hat{\varepsilon} \boldsymbol{E}_{\boldsymbol{k}}-k^{2} \boldsymbol{E}_{\boldsymbol{k}}+\boldsymbol{k}\left(\boldsymbol{k} \cdot \boldsymbol{E}_{\boldsymbol{k}}\right)\right]} \\
& \quad=-\frac{4 \pi q_{0}^{2} d^{2} \Phi_{k}}{\hbar\left(\omega_{0}-\omega\right)} \int \frac{d^{3} k^{\prime}}{(2 \pi)^{3}} \boldsymbol{E}_{k^{\prime}} \Phi_{k^{\prime}}
\end{aligned}
$$

where

$$
\Phi_{k}=\int d^{3} r e^{-i \boldsymbol{k} \cdot \boldsymbol{r}} \Phi(r)=e^{-k^{2} a^{2} / 2}
$$

In the derivation of Eq. (9), we took into account that the function $\Phi_{k}$ depends only on the absolute value of the vector $\boldsymbol{k}$. Equation (9) can be rewritten as

$$
\boldsymbol{E}_{\boldsymbol{k}}=-\frac{4 \pi q_{0}^{2} d^{2}}{\omega_{0}-\omega} \Phi_{k} \hat{G}_{\boldsymbol{k}} \boldsymbol{\Lambda}
$$

where we introduced the Green function in the $\boldsymbol{k}$ space

$$
\hat{G}_{k}=\left(\hat{M}_{k}\right)^{-1}, \quad M_{k, \alpha \beta}=q_{0}^{2} \varepsilon_{\alpha \beta}-k^{2} \delta_{\alpha \beta}+k_{\alpha} k_{\beta},
$$

and defined a new variable $\boldsymbol{\Lambda}=\int \boldsymbol{E}_{\boldsymbol{k}} \Phi_{k} d^{3} k /(2 \pi)^{3}$. Multiplying both parts of Eq. (9) by $\Phi_{k}$ and integrating over $\boldsymbol{k}$, we obtain the matrix equation for the complex eigenfrequencies $\omega$ :

$$
\left(\omega-\omega_{0}\right) \boldsymbol{\Lambda}=\hat{R} \boldsymbol{\Lambda}, \quad \hat{R}=\frac{4 \pi q_{0}^{2} d^{2}}{\hbar} \int \frac{d^{3} k}{(2 \pi)^{3}} \Phi_{k}^{2} \hat{G}_{\boldsymbol{k}} .
$$

We note that the matrix $\hat{R}$ on the right-hand side of Eq. (13) generally depends on the frequency $\omega$. However, we are interested in the weak-coupling regime, when the interaction of the emitter with light can be treated as a perturbation [42], and we set $\hat{R}(\omega)=\hat{R}\left(\omega_{0}\right)$ in Eq. (13). Taking into account that the matrix $\hat{R}$ is diagonal due to the symmetry of the problem, we obtain the spontaneous emission times

$$
\frac{1}{\tau_{\alpha}}=-\frac{8 \pi d^{2} q_{0}^{2}}{\hbar} \int \frac{d^{3} k}{(2 \pi)^{3}} \operatorname{Im} G_{k, \alpha \alpha} \Phi_{k}^{2}, \quad \alpha=x, y, z .
$$

The times $\tau_{x}=\tau_{y}$ and $\tau_{z}$ describe the decay of the source, initially polarized in the plane $x y$ and along the $z$ axis, respectively. To find the decay rates, one should substitute the explicit expressions for the Green function,

$$
\begin{gathered}
G_{\boldsymbol{k}, z z}=\frac{1}{\varepsilon_{\|}} \frac{1-k_{z}^{2} /\left(q_{0}^{2} \varepsilon_{\perp}\right)}{q_{0}^{2}-k_{\perp}^{2} / \varepsilon_{\|}-k_{\|}^{2} / \varepsilon_{\perp}}, \\
G_{\boldsymbol{k}, x x}=\frac{1}{k_{\perp}^{2} \varepsilon_{\perp}}\left\{\frac{k_{y}^{2}}{q_{0}^{2}-k^{2} / \varepsilon_{\perp}}+\frac{k_{x}^{2}\left[1-k_{\perp}^{2} /\left(q_{0}^{2} \varepsilon_{\|}\right)\right]}{q_{0}^{2}-k_{\perp}^{2} / \varepsilon_{\|}-k_{\|}^{2} / \varepsilon_{\perp}}\right\},
\end{gathered}
$$

into Eq. (14).

\section{RESULTS AND DISCUSSION}

The huge Purcell factor in a hyperbolic medium is attained due to the peculiar density of states [14]. To illustrate this effect let us analyze the general structure of Eqs. (14) and (15). As follows from Eq. (15), the axial dipole couples both with transverse electric (TE, ordinary) and transverse magnetic (TM, extraordinary) waves, while the orthogonal dipole couples only with TM waves. Mode dispersion can be determined from the poles of the Green functions and, for the TM waves, it reads

$$
\frac{\omega_{\mathrm{TM}}^{2}}{c^{2}}=\frac{k_{\perp}^{2}}{\varepsilon_{\|}}+\frac{k_{\|}^{2}}{\varepsilon_{\perp}} .
$$

In the case of vanishing losses, $\varepsilon_{\|}^{\prime \prime} \rightarrow 0, \varepsilon_{\perp}^{\prime \prime} \rightarrow 0$ the contributions to the imaginary part of the Green functions that determine the radiative decay rate (14) are determined only by the poles at the eigenmodes. In particular, the TM-mode 
contribution to the rate can be presented in the form

$$
\frac{1}{\tau_{\alpha}^{\mathrm{TM}}}=\frac{2 \pi}{\hbar} \int \frac{d^{3} k}{(2 \pi)^{3}} f_{\alpha}(\boldsymbol{k}) \Phi_{k}^{2} \delta\left(\hbar \omega_{0}-\hbar \omega_{\mathrm{TM}}(\boldsymbol{k})\right),
$$

where the factor $f_{\alpha}(\boldsymbol{k})$ is proportional to the residue of the integrand of (14) at the pole $\omega=\omega_{\mathrm{TM}}(\boldsymbol{k})$. Equation (17) is just the Fermi golden rule [43]; it shows that the decay rate of the emitter with eigenfrequency $\omega_{0}$ is determined by its interaction with all the modes of the surrounding medium having the same frequency. In the hyperbolic medium, the number of such modes is infinite; that is, the density of TM modes,

$$
\rho_{\mathrm{TM}}(\omega)=\int \frac{d^{3} k}{(2 \pi)^{3}} \delta\left(\hbar \omega_{0}-\hbar \omega_{\mathrm{TM}}(\boldsymbol{k})\right),
$$

diverges. Indeed, for $\varepsilon_{\|} \varepsilon_{\perp}<0$ the integral (18) yields the infinite area of the hyperboloid (16); see also insets in Figs. 1(a) and $1(\mathrm{~b})$. However, the radiative rate still remains finite because the interaction with high- $k$ modes is suppressed by the factor $\Phi_{k}^{2}$, assuring the convergence of integrals (14) and (17). The maximum wave vector of the mode contributing to the decay rate is on the order of the inverse size of the emitter; $k_{\max }=1 / a$. Thus, although the specific choice of the function $\Phi_{k}$ does not qualitatively influence our results, the nonlocal character of the material relation (5), arising from the finite spatial extent of the source, is essential to obtain finite results for the decay rate. We note that the cutoff at wave vector $k \sim 1 / a$ is known and exploited, for instance, in the nonrelativistic theory of the Lamb shift [44]. The realistic metamaterial such as the wire medium is characterized by the lattice constant $a_{0}$. If the emitter size is smaller than the spacing between the wires (i.e., $a<a_{0}$ ), our approach is not applicable, and the cutoff is provided at $k_{\max } \sim 1 / a_{0}$. It was shown in Ref. [45] that the enhancement of the density of transverse electromagnetic (TEM) modes in the wire medium as compared to the TM modes in vacuum is on the order of $1 /\left(q_{0} a_{0}\right)^{2}$. This value provides the estimation of the Purcell factor for the wire medium.

Thus, the diverging density of TM modes in the hyperbolic medium leads to the large (but finite) radiative decay rates. Let us now proceed to the quantitative analysis of the Purcell factor. The integral in Eq. (14) can readily be calculated numerically. For the case where the source size smaller than its wavelength, one can also obtain explicit analytical expressions (see the appendix for more details):

$$
\begin{aligned}
\frac{1}{\tau_{z}}= & \frac{q_{0}^{3} d^{2}}{\hbar}\left\{\operatorname{Im} \frac{\arctan \sqrt{\varepsilon-1}-\sqrt{\varepsilon-1}}{\sqrt{\pi} \varepsilon_{\perp}(\varepsilon-1)^{3 / 2}\left(q_{0} a\right)^{3}}\right. \\
& \left.+\operatorname{Im} \frac{(\varepsilon-2) \arctan \sqrt{\varepsilon-1}+\sqrt{\varepsilon-1}}{\sqrt{\pi}(\varepsilon-1)^{3 / 2} q_{0} a}+\frac{4}{3} \operatorname{Re} \sqrt{\varepsilon_{\perp}}\right\}, \\
\frac{1}{\tau_{x}}= & \frac{1}{\tau_{y}}=\frac{q_{0}^{3} d^{2}}{\hbar}\left\{\operatorname{Im} \frac{\sqrt{\varepsilon-1}-\varepsilon \arctan \sqrt{\varepsilon-1}}{2 \sqrt{\pi} \varepsilon_{\perp}(\varepsilon-1)^{3 / 2}\left(q_{0} a\right)^{3}}\right. \\
& \left.+\operatorname{Im} \frac{\varepsilon^{2} \arctan \sqrt{\varepsilon-1}-\varepsilon \sqrt{\varepsilon-1}}{2 \sqrt{\pi}(\varepsilon-1)^{3 / 2} q_{0} a}+\operatorname{Re} \frac{\varepsilon_{\|}+3 \varepsilon_{\perp}}{3 \sqrt{\varepsilon_{\perp}}}\right\},
\end{aligned}
$$

where

$$
\varepsilon=\varepsilon_{\|} / \varepsilon_{\perp}
$$

Equations (19) present the central result of this work. They are valid for arbitrary complex values of $\varepsilon_{\|}$and $\varepsilon_{\perp}$ provided that $q_{0} a \sqrt{\left|\varepsilon_{\|}\right|} \ll 1$ and $q_{0} a \sqrt{\left|\varepsilon_{\perp}\right|} \ll 1$. The experimentally observed decay kinetics of the emitter will be determined by the excitation conditions. For the case where the direction of the dipole moment is fixed and makes an angle $\theta$ with the symmetry axis $z$, the decay will be biexponential, with the initial slope given by

$$
\frac{1}{\tau(\theta)}=\frac{\cos ^{2} \theta}{\tau_{z}}+\frac{\sin ^{2} \theta}{\tau_{x}} .
$$

In the isotropic medium, where $\varepsilon_{\perp}=\varepsilon_{\|}=\kappa$, all the rates (19) reduce to

$$
\frac{1}{\tau}=\frac{4 q_{0}^{3} d^{2}}{3 \hbar} \operatorname{Re} \sqrt{\kappa}-\frac{d^{2}}{3 \hbar \sqrt{\pi} a^{3}} \operatorname{Re} \frac{1}{\kappa} .
$$

In a transparent medium $\left(\kappa^{\prime \prime}=0\right)$ the first term in Eq. (21) reduces to the textbook result for the spontaneous emission rate [43]. The second term describes the energy losses due to the heating of the medium [46,47] similarly as for a dipole placed in a pore in metal [5]. This term controls the decay rate, when the real part of the dielectric constant is negative.

In the anisotropic medium with vanishing losses (i.e., $\varepsilon_{\|}^{\prime \prime}=$ $\varepsilon_{\perp}^{\prime \prime} \rightarrow 0$ ), Eqs. (19) reduce to

$$
\frac{1}{\tau_{\alpha}}=\left\{\begin{array}{lll}
W_{\alpha}^{(0)}, & \varepsilon_{\perp}^{\prime}>0, & \varepsilon_{\|}^{\prime}>0 \\
W_{\alpha}^{(0)}+\frac{W_{\alpha}^{(1)}}{q_{0} a}+\frac{W_{\alpha}^{(3)}}{\left(q_{0} a\right)^{3}}, & \varepsilon_{\perp}^{\prime}>0, & \varepsilon_{\|}^{\prime}<0 \\
-\frac{W_{\alpha}^{(1)}}{q_{0} a}+\frac{W_{\alpha}^{(3)}}{\left(q_{0} a\right)^{3}}, & \varepsilon_{\perp}^{\prime}<0, & \varepsilon_{\|}^{\prime}>0 \\
0, & \varepsilon_{\perp}^{\prime}<0, & \varepsilon_{\|}^{\prime}<0,
\end{array}\right.
$$

where $\alpha=x, y, z$

$$
\begin{gathered}
W_{z}^{(0)}=\frac{4 q_{0}^{3} d^{2} \sqrt{\varepsilon_{\perp}^{\prime}}}{3 \hbar}, \quad W_{x}^{(0)}=\frac{4 q_{0}^{3} d^{2}\left(\varepsilon_{\|}^{\prime}+3 \varepsilon_{\perp}^{\prime}\right)}{3 \hbar \sqrt{\varepsilon_{\perp}^{\prime}}}, \\
W_{z}^{(1)}=-\frac{\sqrt{\pi \mid \varepsilon_{\perp}^{\prime}} \mid q_{0}^{3} d^{2}\left(2\left|\varepsilon_{\perp}^{\prime}\right|+\left|\varepsilon_{\|}^{\prime}\right|\right)}{2 \hbar\left(\left|\varepsilon_{\|}^{\prime}\right|+\left|\varepsilon_{\perp}^{\prime}\right|\right)^{3 / 2}}, \\
W_{x}^{(1)}=\frac{\sqrt{\pi} q_{0}^{3} d^{2}\left|\varepsilon_{\|}^{\prime}\right|^{2}}{4 \hbar \sqrt{\varepsilon_{\perp}^{\prime}}\left(\left|\varepsilon_{\|}^{\prime}\right|+\left|\varepsilon_{\perp}^{\prime}\right|\right)^{3 / 2}}, \\
W_{z}^{(3)}=\frac{\sqrt{\pi\left|\varepsilon_{\perp}^{\prime}\right|} q_{0}^{3} d^{2}}{2 \hbar\left(\left|\varepsilon_{\|}^{\prime}\right|+\left|\varepsilon_{\perp}^{\prime}\right|\right)^{3 / 2}}, \quad W_{x}^{(3)}=\frac{\sqrt{\pi} q_{0}^{3} d^{2}\left|\varepsilon_{\|}^{\prime}\right|}{4 \hbar \sqrt{\left|\varepsilon_{\perp}^{\prime}\right|}\left(\left|\varepsilon_{\|}^{\prime}\right|+\left|\varepsilon_{\perp}^{\prime}\right|\right)^{3 / 2}},
\end{gathered}
$$

and $W_{y} \equiv W_{x}$.

Equation (22) clearly demonstrates nonanalytical behavior with $\varepsilon_{\|}$and $\varepsilon_{\perp}$. When both constants are positive, we deal with traditional uniaxial dielectrics, and transition rates do not depend on the dipole size. For $\varepsilon_{\|}<0$ and $\varepsilon_{\perp}<0$ all radiative rates vanish since the waves in such media are evanescent and do not carry energy away from the source. The most interesting regime occurs when longitudinal and transverse 

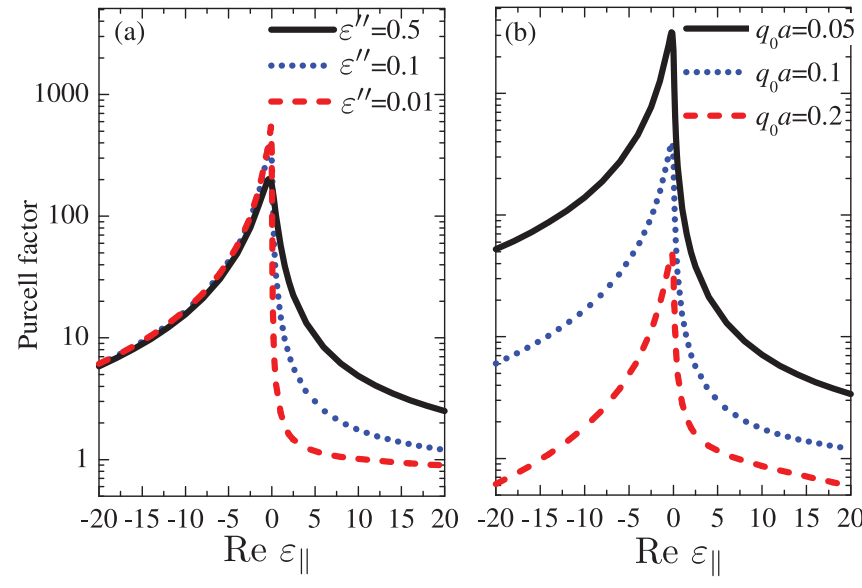

FIG. 2. (Color online) (a) Purcell factor as a function of $\varepsilon_{\|}^{\prime}$ for $\varepsilon_{\perp}^{\prime \prime}=\varepsilon_{\|}^{\prime \prime}=0.01$ (dashed curve), $\varepsilon_{\perp}^{\prime \prime}=\varepsilon_{\|}^{\prime \prime}=0.1$ (dotted curve), $\varepsilon_{\perp}^{\prime \prime}=$ $\varepsilon_{\|}^{\prime \prime}=0.5$ (solid curve), and $q_{0} a=1$. (b) Same as in (a) but for $q_{0} a=0.2$ (dashed curve), $q_{0} a=0.1$ (dotted curve), $q_{0} a=0.05$ (solid curve), and $\varepsilon_{\perp}^{\prime \prime}=\varepsilon_{\|}^{\prime \prime}=0.1$. The dipole is oriented along the $z$ axis.

dielectric constants are of opposite sign $\left(\varepsilon_{\|} \varepsilon_{\perp}<0\right)$. In this case the emission rates are governed by the terms $\propto 1 / a^{3}$ and $\propto 1 / a$ in (22). This result can be understood from the fact that the integrals (14) and (17) are determined by the largest wave vectors, being on the order of $k_{\max } \propto 1 / a$. The same result can be visualized by the real-space emission pattern of the dipole,

$$
E_{\beta}(\boldsymbol{r}) \propto \int \frac{d^{3} k}{(2 \pi)^{3}} e^{i \boldsymbol{k} \cdot \boldsymbol{r}} \Phi_{k} G_{\boldsymbol{k}, \beta \alpha} .
$$

The maximum wave vectors $k_{\max } \sim 1 / a$, contributing to Eq. (24), correspond to the field changing on the spatial scale of the source size $a$. Thus, in the regime when $\varepsilon_{\|} \varepsilon_{\perp}<0$ the radiative emission is determined by the local field effects; see also [17]. The detailed analysis of this peculiar field pattern (24) will be presented elsewhere.

The results of numerical calculation of the transition rates based on Eqs. (14) and (15) are summarized in Figs. 1 to 3. The rates are normalized to their values at $\varepsilon_{\perp}=\varepsilon_{\|}=1$, which yields the Purcell factor with respect to vacuum. Figure 1 shows the Purcell factor for the different dipole orientations as a function of (a) $\varepsilon_{\|}^{\prime}$ and (b) $\varepsilon_{\perp}$. In agreement with Eqs. (19), the rates drastically increase when the real part of one of the dielectric constants becomes negative. Interestingly, the largest enhancement in Fig. 1(a) is achieved when $\varepsilon_{\|}^{\prime}$ is negative but small (i.e., when $\varepsilon_{\|}^{\prime}<0$ and $\left|\varepsilon_{\|}^{\prime}\right| \ll 1$ ). In agreement with this result, the leading terms in Eqs. (22) for the transition rate are proportional to $\sqrt{\varepsilon_{\perp}^{\prime}} /\left(a^{3}\left|\varepsilon_{\|}^{\prime}\right|^{3 / 2}\right)$ for $\left|\varepsilon_{\|}^{\prime}\right| \gg 1$. In addition, this justifies the fact that the observed enhancement is the local field effect because, for large values of $\left|\varepsilon_{\|}^{\prime}\right|$, the local field is screened and so the effect is suppressed. A similar analysis applies for the dependence of the transition rates on $\varepsilon_{\perp}^{\prime}$; see Fig. 1(b). We notice that the analytical results (19) describe all the curves in Fig. 1 with a precision better than $5 \%$.

Figure 2 shows how the Purcell-factor dependence on $\varepsilon_{\|}$ changes (a) with losses and (b) with source size $a$. From Fig. 2(a) we conclude that the losses smear the nonanalytic
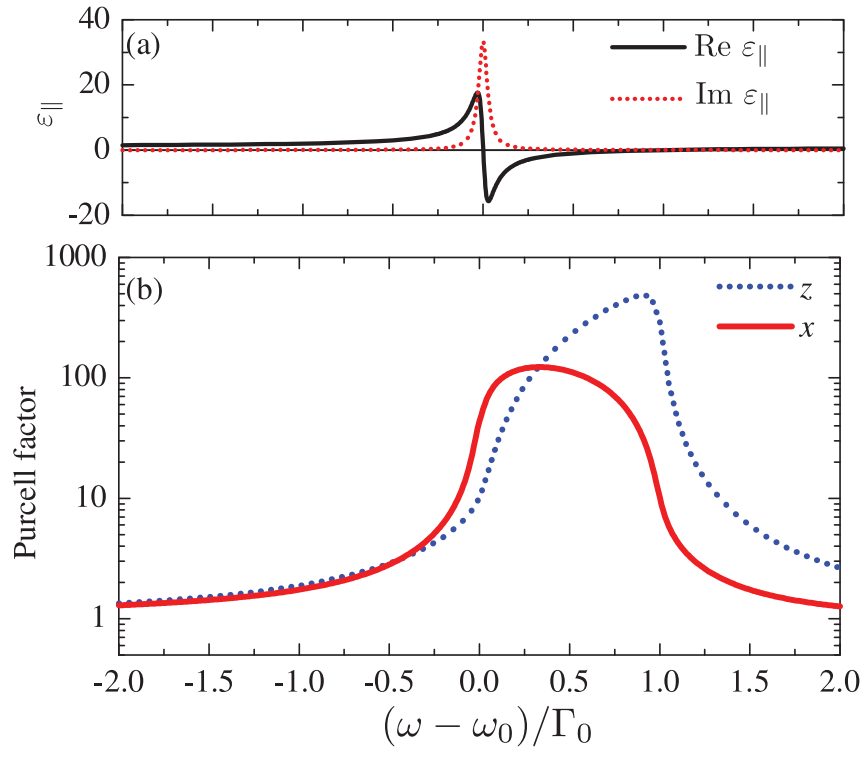

FIG. 3. (Color online) Frequency dependence of the Purcell factor in the medium with $\varepsilon_{\perp}=1$ and $\varepsilon_{\|}$given by Eq. (25). (a) Functions $\varepsilon_{\|}^{\prime}(\omega)$ (solid curve) and $\varepsilon_{\|}^{\prime \prime}(\omega)$ (dotted curve). (b) Purcell factor for the dipoles oriented along $x$ (solid curve) and along $z$ (dotted curve). Calculation was performed for $q_{0} a=0.1, \varepsilon_{\|}^{(0)}=1$, and $\Gamma / \Gamma_{0}=0.03$.

behavior of Purcell factor when $\varepsilon_{\|}^{\prime}$ crosses zero and reduce the maximum value of the Purcell factor. On the other hand, in the regime when $\varepsilon_{\|}^{\prime}>0, \varepsilon_{\perp}^{\prime}>0$, the losses lead to the growth of the decay rate. This is similar to the isotropic case, Eq. (21), and is related to the heating of the medium by the emitted field. Figure 2(b) shows that the Purcell factor is very sensitive to the dipole size. It is quickly suppressed when the size increases, in agreement with the $1 / a^{3}$ and $1 / a$ terms in Eqs. (19).

Figure 3 illustrates the frequency dependence of the Purcell factor in the medium where

$$
\varepsilon_{\|}(\omega)=\varepsilon_{\|}^{(0)}+\frac{\Gamma_{0}}{\omega_{0}-\omega-i \Gamma},
$$

and $\varepsilon_{\perp}=1$. Comparing Figs. 3(a) with 3(b), we observe that the largest enhancement is achieved in the spectral region where $\varepsilon_{\|}^{\prime}(\omega)$ is negative, but small, which agrees with our analysis of Fig. 1. As a result, the positions of the maxima of the curves in Fig. 3(b) are blue shifted from the resonance energy $\omega_{0}$.

\section{CONCLUSION}

We have developed a theory of the Purcell effect for spherical dipole emitters embedded in homogeneous uniaxial media, taking into account the finite size of the emitter and losses in the surrounding medium. We have obtained analytical expressions for the decay rates in the case when the emitter size is much smaller than the wavelength of radiation. We have revealed that, when the real parts of the longitudinal and transverse dielectric constants ( $\varepsilon_{\|}^{\prime}$ and $\varepsilon_{\perp}^{\prime}$, respectively) are of the opposite sign (i.e., for the hyperbolic media), the radiative decay rate depends strongly on the emitter size, and it 
diverges when the size vanishes. This enhancement is related to the divergent density of states in such media and to the peculiar pattern of the local electromagnetic field. The largest Purcell factor is achieved when $\varepsilon_{\|}^{\prime} \varepsilon_{\perp}^{\prime}<0$ and the absolute values of the dielectric constants are much smaller than unity, since the screening of the local electric field in this case is minimal. Our theory has been developed for a simple model of a homogeneous hyperbolic medium. However, due to its general character, it may also provide a qualitative insight into the peculiarities of the spontaneous emission in other types of metamaterials.

\section{ACKNOWLEDGMENTS}

This work was supported by the Ministry of Education and Science of the Russian Federation, RFBR, and the Dynasty Foundation (Russia), EPSRC (UK), and the Australian Research Council. The authors acknowledge illuminating discussions with I. Iorsh, E.L. Ivchenko, S.I. Maslovski, A.S. Potemkin, and C.R. Simovski.

\section{APPENDIX: ANALYTICAL EXPRESSIONS FOR THE DECAY RATES}

In this appendix, we present the details of the derivation of Eqs. (19) .

First, we substitute Eqs. (15) into Eq. (14) and introduce the spherical coordinates $(k, \theta, \varphi)$ in the $\boldsymbol{k}$ space. Integration over the azimuthal angle $d \varphi$ and over $d k$ can be performed analytically and yields

$$
\begin{aligned}
\frac{1}{\tau_{\mathrm{rad}}^{z}}= & \frac{q_{0}^{3} d^{2}}{\hbar} \operatorname{Im} \int_{0}^{\pi} d \theta\left\{-\frac{\chi(\theta) \sin \theta \cos ^{2} \theta}{2 \sqrt{\pi} \varepsilon_{\|} \varepsilon_{\perp}\left(q_{0} a\right)^{3}}+\frac{\chi^{2}(\theta) \sin ^{3} \theta}{\sqrt{\pi} \varepsilon_{\|}^{2} q_{0} a}\right. \\
& \left.+\frac{i \chi^{5 / 2}(\theta) \sin ^{3} \theta}{\varepsilon_{\|}^{2}} e^{-\left(q_{0} a\right)^{2} \chi(\theta)}\left[1+\operatorname{erf}\left(i q_{0} a \sqrt{\chi(\theta)}\right)\right]\right\},
\end{aligned}
$$

$$
\begin{aligned}
\frac{1}{\tau_{\text {rad }}^{x}}= & \frac{1}{\tau_{\text {rad }}^{y}}=\frac{q_{0}^{3} d^{2}}{\hbar} \operatorname{Im} \int_{0}^{\pi} d \theta\left\{\frac{i \sin \theta \sqrt{\varepsilon_{\perp}}}{2} e^{-\varepsilon_{\perp}\left(q_{0} a\right)^{2}}\right. \\
& \times\left[1+\operatorname{erf}\left(i q_{0} a \sqrt{\varepsilon_{\perp}}\right)\right]-\frac{\chi(\theta) \sin ^{3} \theta}{4 \sqrt{\pi} \varepsilon_{\|} \varepsilon_{\perp}\left(q_{0} a\right)^{3}} \\
& +\frac{\sin \theta\left(\varepsilon_{\|}-\chi \sin ^{2} \theta\right)}{2 \sqrt{\pi} \varepsilon_{\|} \varepsilon_{\perp} q_{0} a}+\frac{i \chi^{3 / 2}(\theta) \sin \theta}{2 \varepsilon_{\|} \varepsilon_{\perp}} e^{-\left(q_{0} a\right)^{2} \chi(\theta)} \\
& \left.\times\left[1+\operatorname{erf}\left(i q_{0} a \sqrt{\chi(\theta)}\right)\right]\left[\varepsilon_{\|}-\chi(\theta) \sin ^{2} \theta\right]\right\}
\end{aligned}
$$

where

$$
\frac{1}{\chi(\theta)}=\frac{\sin ^{2} \theta}{\varepsilon_{\perp}}+\frac{\cos ^{2} \theta}{\varepsilon_{\|}},
$$

and the error function is defined as

$$
\operatorname{erf}(x)=\frac{2}{\sqrt{\pi}} \int_{0}^{x} e^{-t^{2}} .
$$

We consider the case when the source size is very small, so that the condition

$$
q_{0} a|\sqrt{\chi(\theta)}| \ll 1, \quad q_{0} a\left|\sqrt{\varepsilon_{\perp}}\right| \ll 1, \quad q_{0} a\left|\sqrt{\varepsilon_{\|}}\right| \ll 1
$$

is satisfied for all values of $\theta$. If the real parts of the dielectric constants have the same sign, the conditions (A4) are easily satisfied for small $q_{0} a$. However, if $\varepsilon_{\|}^{\prime} \varepsilon_{\perp}^{\prime}<0$ the quantity $\chi(\theta)$ may vanish. In this case, the first condition (A4) will still be satisfied provided the imaginary parts of the dielectric constants are sufficiently high. Under the conditions (A4) the exponential functions in Eqs. (A1) can be replaced by unity, and the error functions can be neglected.

After that simplification, the integration over $\theta$ can be performed analytically, and it gives Eqs. (19). Our numerical analysis shows that Eqs. (19) hold even when $\varepsilon_{\perp}^{\prime \prime}, \varepsilon_{\|}^{\prime \prime}$ vanish, provided that the last two conditions (A4) remain valid. In this case, the terms in Eqs. (A1) and (A2) proportional to $\exp \left[-\left(q_{0} a\right)^{2} \chi(\theta)\right]$ are rapidly oscillating, so that their contribution to the integrals becomes small.
[1] E. M. Purcell, Phys. Rev. 69, 681 (1946).

[2] K. Tanaka, E. Plum, J. Y. Ou, T. Uchino, and N. I. Zheludev, Phys. Rev. Lett. 105, 227403 (2010).

[3] T. V. Shubina et al., Phys. Rev. B 82, 073304 (2010).

[4] N. Meinzer, M. Ruther, S. Linden, C. M. Soukoulis, G. Khitrova, J. Hendrickson, J. D. Olitzky, H. M. Gibbs, and M. Wegener, Opt. Express 18, 24140 (2010).

[5] M. M. Glazov, E. L. Ivchenko, A. N. Poddubny, and G. Khitrova, e-print arXiv:1103.6124 (to be published); FTT 53, 1665 (2011) (in Russian).

[6] G. Khitrova, H. M. Gibbs, M. Kira, S. W. Koch, and A. Scherer, Nature Phys. 2, 81 (2006).

[7] A. Dousse, J. Suffczynski, A. Beveratos, O. Krebs, A. Lemaitre, I. Sagnes, J. Bloch, P. Voisin, and P. Senellart, Nature (London) 466, 217 (2010).

[8] S. K. Özdemir, J. Zhu, L. He, and L. Yang, Phys. Rev. A 83, 033817 (2011).
[9] H. Xie, P. Leung, and D. Tsai, Solid State Commun. 149, 625 (2009).

[10] P. Yao, C. Van Vlack, A. Reza, M. Patterson, M. M. Dignam, and S. Hughes, Phys. Rev. B 80, 195106 (2009).

[11] Z. Jacob, J. Kim, G. V. Naik, A. Boltasseva, E. E. Narimanov, and V. M. Shalaev, Appl. Phys. B 100, 215 (2010).

[12] L. V. Alekseyev, E. E. Narimanov, T. Tumkur, H. Li, Y. A. Barnakov, and M. A. Noginov, Appl. Phys. Lett. 97, 131107 (2010).

[13] M. A. Noginov, H. Li, Y. A. Barnakov, D. Dryden, G. Nataraj, G. Zhu, C. E. Bonner, M. Mayy, Z. Jacob, and E. E. Narimanov, Opt. Lett. 35, 1863 (2010).

[14] Z. Jacob, I. Smolyaninov, and E. Narimanov, e-print arXiv:0910.3981 (to be published).

[15] I. V. Lindell, S. A. Tretyakov, K. I. Nikoskinen, and S. Ilvonen, Microwave and Optical Technology Lett. 31, 129 (2001).

[16] D. R. Smith and D. Schurig, Phys. Rev. Lett. 90, 077405 (2003). 
[17] L. Felsen and N. Marcuvitz, Radiation and Scattering of Waves (Wiley Interscience, New York, 2003).

[18] D. R. Smith, D. Schurig, J. J. Mock, P. Kolinko, and P. Rye, Appl. Phys. Lett. 84, 2244 (2004).

[19] D. R. Smith, P. Kolinko, and D. Schurig, J. Opt. Soc. Am. B 21, 1032 (2004).

[20] W. Cai, U. K. Chettiar, A. V. Kildishev, and V. M. Shalaev, Opt. Express 16, 5444 (2008).

[21] P. A. Belov and Y. Hao, Phys. Rev. B 73, 113110 (2006).

[22] A. Salandrino and N. Engheta, Phys. Rev. B 74, 075103 (2006).

[23] Z. Jacob, L. V. Alekseyev, and E. Narimanov, Opt. Express 14, 8247 (2006).

[24] J. Elser, R. Wangberg, V. A. Podolskiy, and E. E. Narimanov, Appl. Phys. Lett. 89, 261102 (2006).

[25] J. Yao, Z. Liu, Y. Liu, Y. Wang, C. Sun, G. Bartal, A. M. Stacy, and X. Zhang, Science 321, 930 (2008).

[26] M. A. Noginov, Y. A. Barnakov, G. Zhu, T. Tumkur, H. Li, and E. E. Narimanov, Appl. Phys. Lett. 94, 151105 (2009).

[27] I. Nefedov, S. Tretyakov, and C. Simovski, e-print arXiv:1102.5263 (to be published).

[28] G. W. Milton, The Theory of Composites (Cambridge University Press, Cambridge, 2004).

[29] P. A. Belov, R. Marqués, S. I. Maslovski, I. S. Nefedov, M. Silveirinha, C. R. Simovski, and S. A. Tretyakov, Phys. Rev. B 67, 113103 (2003).

[30] J. Elser, V. A. Podolskiy, I. Salakhutdinov, and I. Avrutsky, Appl. Phys. Lett. 90, 191109 (2007).
[31] J. Elser, A. A. Govyadinov, I. Avrutsky, I. Salakhutdinov, and V. A. Podolskiy, J. Nanomaterials 2007, 79469 (2007).

[32] J. Elser and V. A. Podolskiy, Phys. Rev. Lett. 100, 066402 (2008).

[33] A. A. Orlov, P. M. Voroshilov, P. A. Belov, and Y. S. Kivshar, e-print arXiv:1103.3847 (to be published).

[34] M. Ya. Azbel and E. A. Kaner, Zh. Exp. Teor. Phys. 30, 811 (1956) [Sov. Phys. JETP 3, 772 (1956)].

[35] R. K. Fisher and R. W. Gould, Phys. Rev. Lett. 22, 1093 (1969).

[36] S. Zhang, Y. Xiong, G. Bartal, X. Yin, and X. Zhang, Phys. Rev. Lett. 106, 243901 (2011).

[37] L. Pilozzi, A. D’Andrea, and K. Cho, Phys. Rev. B 69, 205311 (2004)

[38] E. L. Ivchenko, Optical Spectroscopy of Semiconductor Nanostructures (Alpha Science International, Harrow, 2005).

[39] L. Banyai and S. W. Koch, Semiconductor Quantum Dots (World Scientific, Singapore, 1993).

[40] A. Thränhardt, C. Ell, G. Khitrova, and H. M. Gibbs, Phys. Rev. B 65, 035327 (2002).

[41] S. V. Goupalov, Phys. Rev. B 68, 125311 (2003).

[42] A. Kavokin, J. Baumberg, G. Malpuech, and F. Laussy, Microcavities (Clarendon Press, Oxford, 2006).

[43] A. A. Orlov, P. M. Voroshilov, P. A. Belov, and Y. S. Kivshar, Phys. Rev. B 84, 045424 (2011).

[44] C.-K. Au and G. Feinberg, Phys. Rev. A 9, 1794 (1974).

[45] S. I. Maslovski and M. G. Silveirinha, Phys. Rev. A 83, 022508 (2011).

[46] S. M. Barnett, B. Huttner, R. Loudon, and R. Matloob, J. Phys. B 29, 3763 (1996).

[47] M. S. Tomaš and Z. Lenac, Phys. Rev. A 60, 2431 (1999). 